\title{
A Control Problem for a Heat Conducting Micropolar Fluid
}

\author{
E. Ortega-Torres ${ }^{1}$ \\ Universidad Católica del Norte, Antofagasta, Chile \\ Fernando Vásquez ${ }^{2}$ \\ Universidad Católica del Norte, Antofagasta, Chile
}

\begin{abstract}
We study an optimal control problem associated with the thermally coupled micropolar fluid equations including solid media. The existence of optimal solutions is proved. First order optimality conditions is studied, and an optimality system is derived.
\end{abstract}

Keywords. Micropolar fluids, Boundary control problem, Temperature control.

\section{Introduction}

Let $\Omega \subset \mathbb{R}^{2}$ a bounded open set with boundary $\Gamma$, and suppose $\Omega$ consists of two disjoint subdomains $\Omega_{f}$ and $\Omega_{s}$ where $\Omega_{f}$ represent the fluid domain and $\Omega_{s}$ the solid domain. The boundary of $\Gamma$ is considered as $\Gamma=\Gamma_{1} \cup \Gamma_{2}$ where $\Gamma_{1}=\Gamma \cap \bar{\Omega}_{f}$ and $\Gamma_{2}=\Gamma \cap \bar{\Omega}_{s}$. Also, $\partial \Omega_{f}=\Gamma_{1} \cup \Gamma_{0}$ and $\partial \Omega_{s}=\Gamma_{2} \cup \Gamma_{0}$ where $\Gamma_{0}=\bar{\Omega}_{f} \cap \bar{\Omega}_{s}$. Let $\boldsymbol{u}, w$ and $p$ denote the restriction of the velocity vector, the microrotational velocity and the pressure to the fluid domain $\Omega_{f}$, respectively, since $\boldsymbol{u} \equiv \mathbf{0}$ and $w \equiv 0$ in the solid domain $\Omega_{s}$. Let $\theta_{1}$ and $\theta_{2}$ the restriction of the temperature to the fluid domain $\Omega_{f}$ and solid domain $\Omega_{s}$, respectively; which by continuity of temperature must satisfy the condition $\theta_{1}=\theta_{2}$ on $\Gamma_{0}$. Then, the equations describing the motion of a viscous incompressible stationary micropolar fluid with heat diffusion are given by

$$
\begin{aligned}
-\left(\nu+\nu_{r}\right) \Delta \boldsymbol{u}+\boldsymbol{u} \cdot \nabla \boldsymbol{u}+\nabla p+\alpha \mathbf{G} \theta_{1} & =2 \nu_{r} \operatorname{rot} w+\boldsymbol{f} \quad \text { in } \Omega_{f}, \\
-\left(c_{a}+c_{d}\right) \Delta w+\boldsymbol{u} \cdot \nabla w+4 \nu_{r} w & =2 \nu_{r} \operatorname{rot} \boldsymbol{u}+g \quad \text { in } \Omega_{f}, \\
\operatorname{div} \boldsymbol{u} & =0 \quad \text { in } \Omega_{f}, \\
-\kappa_{1} \Delta \theta_{1}+\boldsymbol{u} \cdot \nabla \theta_{1} & =h_{1} \quad \text { in } \Omega_{f}, \\
-\kappa_{2} \Delta \theta_{2} & =h_{2} \quad \text { in } \Omega_{s}, \\
\boldsymbol{u}=\boldsymbol{g}_{1}, \quad w=0 \quad \theta_{1} & =\eta_{1} \quad \text { on } \Gamma_{1}, \\
\boldsymbol{u}=\mathbf{0}, \quad w & =0 \quad \text { on } \Gamma_{0}, \\
\theta_{2} & =\eta_{2} \quad \text { on } \Gamma_{2}, \\
\kappa_{1} \frac{\partial \theta_{1}}{\partial \boldsymbol{n}_{1}}+\kappa_{2} \frac{\partial \theta_{2}}{\partial \boldsymbol{n}_{2}} & =0 \quad \text { on } \Gamma_{0},
\end{aligned}
$$

\footnotetext{
${ }^{1}$ eortega@ucn.cl

${ }^{2}$ fvasquez@ucn.cl, partially supported by Grant-Conicyt 21161291
} 
where the functions $\boldsymbol{f}$ and $g$ denote external forces actuating on the fluid. The functions $h_{1}$ and $h_{2}$ denote heat sources in the fluid domain $\Omega_{f}$ and solid domain $\Omega_{s}$, respectively. The function $\mathbf{G}$ is the gravity acceleration and $\alpha>0$ is a constant associated with the volume expansion coefficient. The positive constants $\nu, \nu_{r}, \kappa_{1}$ and $\kappa_{2}$ represent the cinematic viscosity of the fluid, the cinematic viscosity of microrotation, the thermic conductivity coefficients in $\Omega_{f}$ and $\Omega_{s}$, respectively. The positive constants $c_{a}$ and $c_{d}$ characterize isotropic properties of the fluid, and are coefficients of angular viscosities; $\boldsymbol{n}_{1}$ and $\boldsymbol{n}_{2}$ denote the exterior unit normal vector to $\Omega_{f}$ and $\Omega_{s}$, respectively, and satisfy $\boldsymbol{n}_{1}=-\boldsymbol{n}_{2}$ on the interface $\Gamma_{0}$. The functions $\boldsymbol{g}_{1}, \eta_{1}$ and $\eta_{2}$ are prescribed functions on the respective boundary. More details of the model (1)-(4) can be seen in [4].

For the condition (9), it is introduced a new variable $g_{2}$ such that

$$
g_{2}=\kappa_{1} \frac{\partial \theta_{1}}{\partial \boldsymbol{n}_{1}}, \quad-g_{2}=\kappa_{2} \frac{\partial \theta_{2}}{\partial \boldsymbol{n}_{2}} \text { on } \Gamma_{0}
$$

Since an arbitrary $g_{2}$ does not guarantee the condition of continuity $\theta_{1}-\theta_{2}=0$ on the interface $\Gamma_{0}$, we consider a problem control in which we want find optimal functions $\boldsymbol{g}_{1}$ and $g_{2}$ defined on $\Gamma_{1}$ and $\Gamma_{0}$, respectively; such that minimize the $L^{2}$ distance of the difference $\theta_{1}-\theta_{2}$ along the interface $\Gamma_{0}$.

In the case of the Boussinesq equations for natural convection with boundary conditions $\boldsymbol{g}_{1}=\mathbf{0}$ on $\Gamma_{1}, \eta_{1}=0$ on $\Gamma_{1}, \eta_{2}=0$ on $\Gamma_{0}$ and (9), the existence of weak solutions has been studied in [3]. An optimal control problem for the thermally coupled incompressible Navier - Stokes equations by Neumann boundary heat control is considered in $[2,3]$.

In section 2 we present existence and uniqueness result for the state equations (1)-(9). In section 3 deals with a boundary control problem that minimize $\left\|\theta_{1}-\theta_{2}\right\|_{L^{2}\left(\Gamma_{0}\right)}$. In section 4 first order necessary optimality conditions are obtained.

\section{Existence of Solutions}

We consider Sobolev space $\mathbf{H}^{1}(\Omega)$ with the usual inner product and norm, and we define the functions spaces $H_{0}^{1}(\Omega)=\left\{w \in H^{1}(\Omega): w=0\right.$ on $\left.\Gamma\right\}$ with norm $\|w\|_{H_{0}^{1}}=\|\nabla w\|_{L^{2}}$, $\mathbf{V}_{f}=\left\{\boldsymbol{u} \in \mathbf{H}_{0}^{1}\left(\Omega_{f}\right): \operatorname{div} \boldsymbol{u}=0\right.$ in $\left.\Omega_{f}\right\}$ with norm $\|\boldsymbol{u}\|_{\mathbf{V}_{f}}=\|\nabla \boldsymbol{u}\|_{L^{2}}, W_{1}=\left\{\theta \in H^{1}\left(\Omega_{f}\right):\right.$ $\theta=0$ on $\left.\Gamma_{1}\right\}$ with norm $\|\theta\|_{W_{1}}=\|\nabla \theta\|_{L^{2}}, W_{2}=\left\{\vartheta \in H^{1}\left(\Omega_{s}\right): \vartheta=0\right.$ on $\left.\Gamma_{2}\right\}$ with norm $\|\vartheta\|_{W_{2}}=\|\nabla \vartheta\|_{L^{2}}, \mathbf{H}_{\sigma}^{1}=\left\{\boldsymbol{u} \in \mathbf{H}^{1}\left(\Omega_{f}\right): \operatorname{div} \boldsymbol{u}=0\right.$ in $\Omega_{f}, \boldsymbol{u} \cdot \boldsymbol{n}=0$ on $\left.\Gamma_{1} \cup \Gamma_{0}\right\}$ with norm $\|\boldsymbol{u}\|_{\mathbf{H}_{\sigma}^{1}}=\|\boldsymbol{u}\|_{\mathbf{H}^{1}}$. For $\Gamma_{k}$ a connected subset of the boundary $\Gamma$, we define the trace spaces $H_{0}^{1 / 2}\left(\Gamma_{k}\right)=\left\{\phi \in L^{2}\left(\Gamma_{\sigma}\right): \exists \hat{\phi} \in H^{1 / 2}(\Gamma), \hat{\phi}_{\left.\right|_{\Gamma_{k}}}=\phi, \hat{\phi}_{\left.\right|_{\Gamma \backslash \Gamma_{k}}}=0\right\}, \mathbf{H}_{00}^{1 / 2}\left(\Gamma_{k}\right)=\left\{\boldsymbol{u} \in \mathbf{L}^{2}\left(\Gamma_{\sigma}\right):\right.$ $\left.\exists \boldsymbol{v} \in \mathbf{H}^{1 / 2}(\Gamma), \boldsymbol{v}_{\left.\right|_{\Gamma_{k}}}=\boldsymbol{u}, \boldsymbol{v}_{\left.\right|_{\Gamma \backslash \Gamma_{k}}}=\mathbf{0}, \int_{\Gamma_{k}} \boldsymbol{u} \cdot \boldsymbol{n} d \Gamma=0\right\}$. Also, by simplicity we define the product spaces $\mathbb{H}=\mathbf{H}_{\sigma}^{1} \times H_{0}^{1}\left(\Omega_{f}\right) \times H^{1}\left(\Omega_{f}\right) \times H^{1}\left(\Omega_{s}\right), \mathbb{X}=\mathbf{V}_{f} \times H_{0}^{1}\left(\Omega_{f}\right) \times W_{1} \times W_{2}$, with the usual norms. The dual space of $\mathbb{X}$ is given by $\mathbb{X}^{\prime}=\mathbf{V}_{f}^{\prime} \times H^{-1}\left(\Omega_{f}\right) \times W_{1}^{\prime} \times W_{2}^{\prime}$.

By using integration by part and (10), a weak formulation for the system (1)-(9) is: 
Find $\left(\boldsymbol{u}, w, \theta_{1}, \theta_{2}\right) \in \mathbb{H}$ such that

$$
\begin{aligned}
\nu_{1}(\nabla \boldsymbol{u}, \nabla \boldsymbol{\varphi})+(\boldsymbol{u} \cdot \nabla \boldsymbol{u}, \boldsymbol{\varphi})+\alpha\left(\mathbf{G} \theta_{1}, \boldsymbol{\varphi}\right) & =2 \nu_{r}(\operatorname{rot} w, \boldsymbol{\varphi})+\langle\boldsymbol{f}, \boldsymbol{\varphi}\rangle \quad \forall \boldsymbol{\varphi} \in \mathbf{V}_{f} \\
\nu_{2}(\nabla w, \nabla \psi)+(\boldsymbol{u} \cdot \nabla w, \psi)+4 \nu_{r}(w, \psi) & =2 \nu_{r}(\operatorname{rot} \boldsymbol{u}, \psi)+\langle g, \psi\rangle \quad \forall \psi \in H_{0}^{1}\left(\Omega_{f}\right), \\
\kappa_{1}\left(\nabla \theta_{1}, \nabla \phi\right)+\left(\boldsymbol{u} \cdot \nabla \theta_{1}, \phi\right) & =\left\langle h_{1}, \phi\right\rangle+\left(g_{2}, \phi\right)_{\Gamma_{0}} \quad \forall \phi \in W_{1} \\
\kappa_{2}\left(\nabla \theta_{2}, \nabla \xi\right) & =\left\langle h_{2}, \xi\right\rangle-\left(g_{2}, \xi\right)_{\Gamma_{0}} \quad \forall \xi \in W_{2} \\
\boldsymbol{u} & =\boldsymbol{u}_{\boldsymbol{g}_{1}} \text { on } \Gamma_{1} \cup \Gamma_{0} \\
\theta_{1} & =\eta_{1} \quad \text { on } \Gamma_{1} \\
\theta_{2} & =\eta_{2} \quad \text { on } \Gamma_{2}
\end{aligned}
$$

where $\boldsymbol{u}_{\boldsymbol{g}_{1}}=\boldsymbol{g}_{1}$ on $\Gamma_{1}, \boldsymbol{u}_{\boldsymbol{g}_{1}}=\mathbf{0}$ on $\Gamma_{0}, \nu_{1}=\nu+\nu_{r}$ and $\nu_{2}=c_{a}+c_{d}$.

About the existence of solutions of the system (11)-(17) we have the following result.

Theorem 2.1. Let $\left(\boldsymbol{f}, g, h_{1}, h_{2}\right) \in \mathbb{X}^{\prime}, \boldsymbol{G} \in \boldsymbol{L}^{\infty}\left(\Omega_{f}\right), \boldsymbol{g}_{1} \in \boldsymbol{H}_{00}^{1 / 2}\left(\Gamma_{1}\right), \eta_{1} \in H_{0}^{1 / 2}\left(\Gamma_{1}\right)$ and $\eta_{2} \in H_{0}^{1 / 2}\left(\Gamma_{2}\right)$. If $\nu_{1}, \nu_{2}$ and $\kappa_{1}$ are large enough such that

$$
\delta=\min \left\{\nu_{1}-2 \nu_{r} C-C\left\|\eta_{1}\right\|_{H^{1 / 2}\left(\Gamma_{1}\right)}, \nu_{2}-2 \nu_{r} C, \kappa_{1}-\alpha C\|\boldsymbol{G}\|_{\infty}, \kappa_{2}\right\}>0
$$

with $C$ a positive constant, there exists at least one solution $\left(\boldsymbol{u}, w, \theta_{1}, \theta_{2}\right) \in \mathbb{H}$ for the problem (11)-(17). Moreover, the solution satisfies the following inequality

$$
\|\boldsymbol{u}\|_{\boldsymbol{H}_{\sigma}^{1}}+\|w\|_{H_{0}^{1}}+\left\|\theta_{1}\right\|_{H^{1}}+\left\|\theta_{2}\right\|_{H^{1}} \leq C_{1} \Theta
$$

where $C_{1}>0$ is a constant depending on $\nu_{1}, \nu_{r}, \nu_{2}, \kappa_{1}, \kappa_{2}$ and $\delta, \Theta>0$ is a constant depending on $\left\|\boldsymbol{u}_{\boldsymbol{g}_{1}}\right\|_{\Gamma_{1} \cup \Gamma_{0}},\left\|\eta_{1}\right\|_{H^{1 / 2}\left(\Gamma_{1}\right)},\left\|\eta_{2}\right\|_{H^{1 / 2}\left(\Gamma_{2}\right)},\|\boldsymbol{G}\|_{\infty},\|\boldsymbol{f}\|_{\boldsymbol{V}_{f}^{\prime}},\|g\|_{H^{-1}},\left\|h_{1}\right\|_{W_{1}^{\prime}},\left\|h_{2}\right\|_{W_{2}^{\prime}}$ and $\left\|g_{2}\right\|_{L^{2}\left(\Gamma_{0}\right)}$.

Proof. Follows by using the Lax-Milgram lemma and Leray-Schauder fixed point theorem.

About the uniqueness of the solution, a result is given in the following theorem.

Theorem 2.2. Under the conditions of the Theorem 2.1, if $\delta>C_{1} \Theta$ the solution of the problem (11)-(17) is unique.

\section{Boundary Control Problem}

As controls spaces we consider the closed convex sets $\mathcal{U}_{1} \subset \mathbf{H}_{00}^{1 / 2}\left(\Gamma_{1}\right)$ and $\mathcal{U}_{2} \subset L^{2}\left(\Gamma_{0}\right)$, then the functions $\boldsymbol{g}=\left(\boldsymbol{g}_{1}, g_{2}\right) \in \mathcal{U}_{1} \times \mathcal{U}_{2}=\mathcal{U}_{a d}$ represent boundary controls, being $\boldsymbol{g}_{1}$ a boundary control for the velocity on the part $\Gamma_{1}$ of the boundary of $\Omega_{f}$ and $g_{2}$ a control for the temperature on the interface $\Gamma_{0}$ of the solid-fluid region. Also, is considered a function $\boldsymbol{u}_{d} \in \mathbf{L}^{2}\left(\Omega_{f}\right)$ which represent a desired velocity for the fluid.

Denoting $\boldsymbol{z}=\left(\boldsymbol{u}, w, \theta_{1}, \theta_{2}\right) \in \mathbb{H}$ and $\boldsymbol{g}=\left(\boldsymbol{g}_{1}, g_{2}\right) \in \mathcal{U}_{a d}$, we establish the following boundary control problem: Find $(\boldsymbol{z}, \boldsymbol{g}) \in \mathbb{H} \times \mathcal{U}_{a d}$ such that minimize the functional

$$
J(\boldsymbol{z}, \boldsymbol{g})=\frac{1}{2}\left\|\boldsymbol{u}-\boldsymbol{u}_{d}\right\|_{\mathbf{L}^{2}\left(\Omega_{f}\right)}^{2}+\frac{1}{2}\left\|\theta_{1}-\theta_{2}\right\|_{L^{2}\left(\Gamma_{0}\right)}^{2}+\frac{\beta_{1}}{2}\left\|\boldsymbol{g}_{1}\right\|_{\mathbf{H}^{1 / 2}\left(\Gamma_{1}\right)}^{2}+\frac{\beta_{2}}{2}\left\|g_{2}\right\|_{L^{2}\left(\Gamma_{0}\right)}^{2}
$$


subject to $(\boldsymbol{z}, \boldsymbol{g})$ satisfying (11)-(17).

The constants $\beta_{1}$ and $\beta_{2}$ satisfy any of the following conditions:

(i) If $\beta_{1} \geq 0$ and $\beta_{2} \geq 0, \mathcal{U}_{1}$ and $\mathcal{U}_{2}$ are bounded closed convex sets.

(ii) If $\beta_{1}>0$ and $\beta_{2}>0, \mathcal{U}_{1}$ and $\mathcal{U}_{2}$ are closed convex sets.

To describe the constraints for the control problem, we define the space

$$
\mathbb{Y}=\mathbb{X}^{\prime} \times \mathbf{H}_{00}^{1 / 2}\left(\partial \Omega_{f}\right) \times H_{0}^{1 / 2}\left(\Gamma_{1}\right) \times H_{0}^{1 / 2}\left(\Gamma_{2}\right)
$$

and we consider the constraint operator $\mathbb{F}=\left(\mathbf{F}_{1}, F_{2}, F_{3}, F_{4}, \mathbf{F}_{5}, F_{6}, F_{7}\right): \mathbb{H} \times \mathcal{U}_{a d} \rightarrow \mathbb{Y}$, such that in each point $\boldsymbol{s}=(\boldsymbol{z}, \boldsymbol{g}) \in \mathbb{H} \times \mathcal{U}_{a d}$ is defined by

$$
\begin{aligned}
\left\langle\mathbf{F}_{1}(\boldsymbol{s}), \boldsymbol{\varphi}\right\rangle_{\mathbf{V}_{f}^{\prime}} & =\nu_{1}(\nabla \boldsymbol{u}, \nabla \boldsymbol{\varphi})+(\boldsymbol{u} \cdot \nabla \boldsymbol{u}, \boldsymbol{\varphi})+\alpha\left(\mathbf{G} \theta_{1}, \boldsymbol{\varphi}\right)-2 \nu_{r}(\operatorname{rot} w, \boldsymbol{\varphi})-\langle\boldsymbol{f}, \boldsymbol{\varphi}\rangle_{\mathbf{V}_{f}^{\prime}}, \\
\left\langle F_{2}(\boldsymbol{s}), \psi\right\rangle_{H^{-1}} & =\nu_{2}(\nabla w, \nabla \psi)+(\boldsymbol{u} \cdot \nabla w, \psi)+4 \nu_{r}(w, \psi)-2 \nu_{r}(\operatorname{rot} \boldsymbol{u}, \psi)-\langle g, \psi\rangle_{H^{-1}}, \\
\left\langle F_{3}(\boldsymbol{s}), \phi\right\rangle_{W_{1}^{\prime}} & =\kappa_{1}\left(\nabla \theta_{1}, \nabla \phi\right)+\left(\boldsymbol{u} \cdot \nabla \theta_{1}, \phi\right)-\left\langle h_{1}, \phi\right\rangle-\left(g_{2}, \phi\right)_{\Gamma_{0}}, \\
\left\langle F_{4}(\boldsymbol{s}), \xi\right\rangle_{W_{2}^{\prime}} & =\kappa_{2}\left(\nabla \theta_{2}, \nabla \xi\right)-\left\langle h_{2}, \xi\right\rangle+\left(g_{2}, \xi\right)_{\Gamma_{0}}, \\
\mathbf{F}_{5}(\boldsymbol{s}) & =\boldsymbol{u}_{\left.\right|_{\Gamma_{0} \cup \Gamma_{1}}}-\boldsymbol{u}_{\boldsymbol{g}_{1}}, \quad F_{6}(\boldsymbol{s})=\theta_{1_{\Gamma_{1}}}-\eta_{1}, \quad F_{7}(\boldsymbol{s})=\theta_{\left.\right|_{\Gamma_{2}}}-\eta_{2},
\end{aligned}
$$

for all $(\varphi, \psi, \phi, \xi) \in \mathbb{X}$.

Then, we reformulate the above control problem as: Find $(\boldsymbol{z}, \boldsymbol{g}) \in \mathbb{H} \times \mathcal{U}_{a d}$ such that minimize the functional

$$
J(\boldsymbol{z}, \boldsymbol{g})=\frac{1}{2}\left\|\boldsymbol{u}-\boldsymbol{u}_{d}\right\|_{\mathbf{L}^{2}\left(\Omega_{f}\right)}^{2}+\frac{1}{2}\left\|\theta_{1}-\theta_{2}\right\|_{L^{2}\left(\Gamma_{0}\right)}^{2}+\frac{\beta_{1}}{2}\left\|\boldsymbol{g}_{1}\right\|_{\mathbf{H}^{1 / 2}\left(\Gamma_{1}\right)}^{2}+\frac{\beta_{2}}{2}\left\|g_{2}\right\|_{L^{2}\left(\Gamma_{0}\right)}^{2}
$$

subject to $\mathbb{F}(\boldsymbol{z}, \boldsymbol{g})=\mathbf{0}$ in $\mathbb{Y}$.

The admissible solutions set for the control problem (20) is defined by

$$
\mathcal{S}_{a d}=\left\{\boldsymbol{s}=(\boldsymbol{z}, \boldsymbol{g}) \in \mathbb{H} \times \mathcal{U}_{a d}: J(\boldsymbol{s})<\infty \text { and satisfies } \mathbb{F}(\boldsymbol{z}, \boldsymbol{g})=\mathbf{0}\right\} .
$$

About the existence of solution for the control problem (20) we give the following result.

Theorem 3.1. Under the conditions of the Theorem 2.1, if $\mathcal{U}_{\text {ad }}$ satisfies any of the conditions (18)-(19), the control problem (20) has at least a solution $\overline{\boldsymbol{s}}=(\overline{\boldsymbol{z}}, \overline{\boldsymbol{g}}) \in \mathcal{S}_{a d}$.

Proof. By Theorem 2.1 the set $\mathcal{S}_{a d}$ is not empty. Since $J(\boldsymbol{s})$ is bounded below, exists a minimizing sequence $\left\{\boldsymbol{s}^{m}=\left(\boldsymbol{u}^{m}, w^{m}, \theta_{1}^{m}, \theta_{2}^{m}, \boldsymbol{g}_{1}^{m}, g_{2}^{m}\right)\right\}_{m \geq 1} \in \mathcal{S}_{a d}$ such that

$$
\lim _{m \rightarrow \infty} J\left(s^{m}\right)=\inf _{s \in \mathcal{S}_{a d}} J(s) .
$$

Moreover, $J\left(s^{m}\right)<\infty$ and $s^{m}$ satisfies the system (11)-(17), then by Theorem 2.1 we obtain that $\left\{s^{m}\right\}$ is bounded in $\mathbb{H} \times \mathcal{U}_{a d}$, and since $\mathcal{U}_{a d}$ is a closed and convex subset of $\mathbf{H}_{00}^{1 / 2}\left(\Gamma_{1}\right) \times L^{2}\left(\Gamma_{0}\right)$, exists $\overline{\boldsymbol{s}} \in \mathbb{H} \times \mathcal{U}_{a d}$ such that as $m \rightarrow \infty, \boldsymbol{s}^{m} \rightarrow \overline{\boldsymbol{s}}$ weakly in $\mathbb{H} \times \mathcal{U}_{a d}$. Moreover, taking into account that the embedding $\mathbf{H}_{\sigma}^{1} \hookrightarrow \mathbf{L}^{2}\left(\Omega_{f}\right), H_{0}^{1}\left(\Omega_{f}\right) \hookrightarrow L^{2}\left(\Omega_{f}\right)$, 
$H^{1}\left(\Omega_{f}\right) \hookrightarrow L^{2}\left(\Omega_{f}\right), H^{1}\left(\Omega_{s}\right) \hookrightarrow L^{2}\left(\Omega_{s}\right)$ and $\mathbf{H}_{00}^{1 / 2}\left(\Gamma_{1}\right) \hookrightarrow \mathbf{L}^{2}\left(\Gamma_{1}\right)$ are compact we deduce that $s^{m} \rightarrow \bar{s}$ strongly in $\mathbf{L}^{2}\left(\Omega_{f}\right) \times L^{2}\left(\Omega_{f}\right) \times L^{2}\left(\Omega_{f}\right) \times L^{2}\left(\Omega_{s}\right) \times \mathbf{L}^{2}\left(\Gamma_{1}\right)$.

Then, by using the above convergences we can obtain that $\bar{s}$ satisfies (11)-(17) and we conclude that $\bar{s} \in \mathcal{S}_{a d}$, which implies

$$
\inf _{\boldsymbol{s} \in \mathcal{S}_{a d}} J(\boldsymbol{s}) \leq J(\bar{s}) .
$$

Since $J$ is weakly lower semi-continuous on $\mathcal{S}_{a d}$ we have that $J(\bar{s}) \leq \lim _{m \rightarrow \infty} \inf J\left(s^{m}\right)$ and then

$$
J(\bar{s}) \leq \lim _{m \rightarrow \infty} J\left(s^{m}\right) .
$$

Now, from (21), (22) and (23) it follows that $\overline{\boldsymbol{s}}$ is a solution for the problem (20).

\section{First Order Necessary Conditions}

For the functional $J$ and the constraint operator $\mathbb{F}$ of the problem (20), we have the following results.

Lemma 4.1. The functional $J$ is Fréchet differentiable with respect to $\boldsymbol{s}=(\boldsymbol{z}, \boldsymbol{g}) \in$ $\mathbb{H} \times \mathcal{U}_{a d}$. Moreover, at the arbitrary point $\tilde{\boldsymbol{s}}=(\tilde{\boldsymbol{z}}, \tilde{\boldsymbol{g}}) \in \mathbb{H} \times \mathcal{U}_{a d}$ the Fréchet derivative of $J$ with respect to $\boldsymbol{s}$ is the linear and bounded functional $J_{\boldsymbol{s}}(\tilde{\boldsymbol{s}}): \mathbb{H} \times \mathcal{U}_{\text {ad }} \rightarrow \mathbb{R}$, such that in each point $\boldsymbol{\tau}=\left(\varrho, \hat{w}, \vartheta_{1}, \vartheta_{2}, \hat{\boldsymbol{g}}_{1}, \hat{g}_{2}\right) \in \mathbb{H} \times \mathcal{U}_{a d}$ is defined by:

$$
J_{\boldsymbol{s}}(\tilde{\boldsymbol{s}}) \boldsymbol{\tau}=\left(\tilde{\boldsymbol{u}}-\boldsymbol{u}_{d}, \varrho\right)+\left(\tilde{\theta}_{1}-\tilde{\theta}_{2}, \vartheta_{1}-\vartheta_{2}\right)_{\Gamma_{0}}+\beta_{1}\left(\tilde{\boldsymbol{g}}_{1}, \hat{\boldsymbol{g}}_{1}\right)_{\Gamma_{1}}+\beta_{2}\left(\tilde{g}_{2}, \hat{g}_{2}\right)_{\Gamma_{0}} .
$$

Lemma 4.2. The operator $\mathbb{F}$ is Fréchet differentiable with respect to $\boldsymbol{s}=(\boldsymbol{z}, \boldsymbol{g}) \in \mathbb{H} \times \mathcal{U}_{\text {ad }}$. Moreover, at the arbitrary point $\tilde{\boldsymbol{s}}=(\tilde{\boldsymbol{z}}, \tilde{\boldsymbol{g}}) \in \mathbb{H} \times \mathcal{U}_{\text {ad }}$ the Fréchet derivative of $\mathbb{F}$ with respect to $\boldsymbol{s}$ is the linear and bounded operator $\mathbb{F}_{\boldsymbol{s}}(\tilde{\boldsymbol{s}}): \mathbb{H} \times \mathcal{U}_{a d} \rightarrow \mathbb{Y}$, such that in each point $\boldsymbol{\tau}=\left(\boldsymbol{\varrho}, \hat{w}, \vartheta_{1}, \vartheta_{2}, \hat{\boldsymbol{g}}_{1}, \hat{g}_{2}\right) \in \mathbb{H} \times \mathcal{U}_{a d}$ is defined by:

$$
\begin{aligned}
& \left\langle\boldsymbol{F}_{1 \boldsymbol{s}}(\tilde{\boldsymbol{s}}) \boldsymbol{\tau}, \boldsymbol{\varphi}\right\rangle_{\boldsymbol{V}_{f}^{\prime}}=\nu_{1}(\nabla \varrho \boldsymbol{\varrho}, \nabla \boldsymbol{\varphi})+(\tilde{\boldsymbol{u}} \cdot \nabla \boldsymbol{\varrho}, \boldsymbol{\varphi})+(\boldsymbol{\varrho} \cdot \nabla \tilde{\boldsymbol{u}}, \boldsymbol{\varphi})+\alpha\left(\boldsymbol{G} \vartheta_{1}, \boldsymbol{\varphi}\right)-2 \nu_{r}(\operatorname{rot} \hat{w}, \boldsymbol{\varphi}), \\
& \left\langle F_{2 \boldsymbol{s}}(\tilde{\boldsymbol{s}}) \boldsymbol{\tau}, \psi\right\rangle_{H^{-1}}=\nu_{2}(\nabla \hat{w}, \nabla \psi)+(\tilde{\boldsymbol{u}} \cdot \nabla \hat{w}, \psi)+(\boldsymbol{\varrho} \cdot \nabla \tilde{w}, \psi)+4 \nu_{r}(\hat{w}, \psi)-2 \nu_{r}(\operatorname{rot} \boldsymbol{\varrho}, \psi) \text {, } \\
& \left\langle F_{3 \boldsymbol{s}}(\tilde{\boldsymbol{s}}) \boldsymbol{\tau}, \phi\right\rangle_{W_{1}^{\prime}}=\kappa_{1}\left(\nabla \vartheta_{1}, \nabla \phi\right)+\left(\tilde{\boldsymbol{u}} \cdot \nabla \vartheta_{1}, \phi\right)+\left(\varrho \cdot \nabla \tilde{\theta}_{1}, \phi\right)-\left(\hat{g}_{2}, \phi\right)_{\Gamma_{0}}, \\
& \left\langle F_{4 \boldsymbol{s}}(\tilde{\boldsymbol{s}}) \boldsymbol{\tau}, \xi\right\rangle_{W_{2}^{\prime}}=\kappa_{2}\left(\nabla \vartheta_{2}, \nabla \xi\right)+\left(\hat{g}_{2}, \xi\right)_{\Gamma_{0}} \\
& \boldsymbol{F}_{5 \boldsymbol{s}}(\tilde{\boldsymbol{s}}) \boldsymbol{\tau}=\varrho_{\Gamma_{\Gamma_{0}} \cup \Gamma_{1}}-B\left(\hat{\boldsymbol{g}}_{1}\right), \quad F_{6 \boldsymbol{s}}(\tilde{\boldsymbol{s}}) \boldsymbol{\tau}=\vartheta_{\left.1\right|_{\Gamma_{1}}}, \quad F_{7 \boldsymbol{s}}(\tilde{\boldsymbol{s}}) \boldsymbol{\tau}=\vartheta_{\left.2\right|_{\Gamma_{2}}},
\end{aligned}
$$

where $B: \boldsymbol{H}_{00}^{1 / 2}\left(\Gamma_{1}\right) \rightarrow \boldsymbol{H}^{1 / 2}\left(\partial \Omega_{f}\right)$ is a linear and bounded operator defined by $B\left(\hat{\boldsymbol{g}}_{1}\right)=\hat{\boldsymbol{g}}_{1}$ on $\Gamma_{1}$ and $B\left(\hat{\boldsymbol{g}}_{1}\right)=\boldsymbol{O}$ on $\Gamma_{0}$.

As in [1] to guarantee the existence of Lagrange multipliers, we establish a condition for a pair $(\boldsymbol{z}, \boldsymbol{g}) \in \mathbb{H} \times \mathcal{U}_{a d}$ to satisfy the regular point condition.

Definition 4.1. ( [5], p. 50) Let $\tilde{\boldsymbol{s}}=(\tilde{\boldsymbol{z}}, \tilde{\boldsymbol{g}}) \in \mathbb{H} \times \mathcal{U}_{a d}$ be a optimal solution of the problem (20). It is said that $\tilde{\boldsymbol{s}}$ satisfy the regular point condition if $\mathbb{F}_{\boldsymbol{s}}(\tilde{\boldsymbol{z}}, \tilde{\boldsymbol{g}})(\mathbb{H} \times \mathcal{C}(\overline{\boldsymbol{g}}))=\mathbb{Y}$, where $\mathcal{C}(\tilde{\boldsymbol{g}})=\mathcal{C}\left(\tilde{\boldsymbol{g}}_{1}\right) \times \mathcal{C}\left(\bar{g}_{2}\right)=\left\{\left(\gamma_{1}\left(\boldsymbol{g}_{1}-\tilde{\boldsymbol{g}}_{1}\right), \gamma_{2}\left(g_{2}-\tilde{g}_{2}\right)\right), \gamma_{1} \geq 0, \gamma_{2} \geq 0,\left(\boldsymbol{g}_{1}, g_{2}\right) \in \mathcal{U}_{a d}\right\}$. 
Denoting $\tilde{\boldsymbol{z}}=\left(\tilde{\boldsymbol{u}}, \tilde{w}, \tilde{\theta}_{1}, \tilde{\theta}_{2}\right), \tilde{\boldsymbol{g}}=\left(\tilde{\boldsymbol{g}}_{1}, \tilde{g}_{2}\right)$, we establish the following result.

Lemma 4.3. Let $\tilde{\boldsymbol{s}}=(\tilde{\boldsymbol{z}}, \tilde{\boldsymbol{g}}) \in \mathcal{S}_{a d}$ be a solution of the problem (20). If $\nu_{1}, \nu_{2}$ and $\kappa_{1}$ are large enough such that

$$
\delta_{1}=\min \left\{\nu_{1}-\alpha C\|\boldsymbol{G}\|_{\infty}-2 C \nu_{r}, \nu_{2}-2 C \nu_{r}, \kappa_{1}-\alpha C\|\boldsymbol{G}\|_{\infty}, \kappa_{2}\right\}>2 C\|\tilde{\boldsymbol{z}}\|_{\mathbb{H}},
$$

with $C$ a positive constant depending only on $\Omega$, then $\tilde{\boldsymbol{s}}$ satisfies the regular point condition. Proof. The steps of the proof are similar to those for the Navier - Stokes equations given in $[1]$.

About the existence of Lagrange multipliers we have the following result.

Theorem 4.1. Let $\tilde{\boldsymbol{s}}=(\tilde{\boldsymbol{z}}, \tilde{\boldsymbol{g}})$ be a local optimal solution for the problem (20) with $\nu_{1}, \nu_{2}$ and $\kappa_{1}$ satisfying (24). Then, there exist Lagrange multipliers

$$
\boldsymbol{\lambda}=\left(\boldsymbol{\lambda}_{1}, \lambda_{2}, \lambda_{3}, \lambda_{4}, \boldsymbol{\lambda}_{5}, \lambda_{6}, \lambda_{7}\right) \in \mathbb{Y}^{\prime}=\mathbb{X} \times \boldsymbol{H}_{00}^{-1 / 2}\left(\partial \Omega_{f}\right) \times H_{0}^{-1 / 2}\left(\Gamma_{1}\right) \times H_{0}^{-1 / 2}\left(\Gamma_{2}\right)
$$

such that for all $\boldsymbol{\tau}=\left(\boldsymbol{\varrho}, \hat{w}, \vartheta_{1}, \vartheta_{2}, \hat{\boldsymbol{g}}_{1}, \hat{g}_{2}\right) \in \mathbb{H} \times \mathcal{C}\left(\tilde{\boldsymbol{g}}_{1}\right) \times \mathcal{C}\left(\tilde{g}_{2}\right)$,

$$
\begin{aligned}
(\tilde{\boldsymbol{u}} & \left.-\boldsymbol{u}_{d}, \varrho\right)+\left(\tilde{\theta}_{1}-\tilde{\theta}_{2}, \vartheta_{1}\right)_{\Gamma_{0}}+\left(\tilde{\theta}_{2}-\tilde{\theta}_{1}, \vartheta_{2}\right)_{\Gamma_{0}}+\beta_{1}\left\langle\tilde{\boldsymbol{g}}_{1}, \hat{\boldsymbol{g}}_{1}\right\rangle_{\Gamma_{1}}+\beta_{2}\left(\tilde{g}_{2}, \hat{g}_{2}\right)_{\Gamma_{0}} \\
& -\nu_{1}\left(\nabla \varrho, \nabla \boldsymbol{\lambda}_{1}\right)-\left(\tilde{\boldsymbol{u}} \cdot \nabla \varrho, \boldsymbol{\lambda}_{1}\right)-\left(\varrho \cdot \nabla \tilde{\boldsymbol{u}}, \boldsymbol{\lambda}_{1}\right)-\alpha\left(\boldsymbol{G} \vartheta_{1}, \boldsymbol{\lambda}_{1}\right)+2 \nu_{r}\left(\operatorname{rot} \hat{w}, \boldsymbol{\lambda}_{1}\right) \\
& -\nu_{2}\left(\nabla \hat{w}, \nabla \lambda_{2}\right)-\left(\tilde{\boldsymbol{u}} \cdot \nabla \hat{w}, \lambda_{2}\right)-\left(\varrho \cdot \nabla \tilde{w}, \lambda_{2}\right)-4 \nu_{r}\left(\hat{w}, \lambda_{2}\right)+2 \nu_{r}\left(\operatorname{rot} \varrho, \lambda_{2}\right) \\
& -\kappa_{1}\left(\nabla \vartheta_{1}, \nabla \lambda_{3}\right)-\left(\tilde{\boldsymbol{u}} \cdot \nabla \vartheta_{1}, \lambda_{3}\right)-\left(\boldsymbol{\varrho} \cdot \nabla \tilde{\theta}_{1}, \lambda_{3}\right)+\left\langle\hat{g}_{2}, \lambda_{3}\right\rangle_{\Gamma_{0}}-\kappa_{2}\left(\nabla \vartheta_{2}, \nabla \lambda_{4}\right) \\
& -\left\langle\hat{g}_{2}, \lambda_{4}\right\rangle_{\Gamma_{0}}-\left\langle\boldsymbol{\lambda}_{5}, \varrho-B\left(\hat{\boldsymbol{g}}_{1}\right)\right\rangle_{\Gamma_{0} \cup \Gamma_{1}}-\left\langle\lambda_{6}, \vartheta_{1}\right\rangle_{\Gamma_{1}}-\left\langle\lambda_{7}, \vartheta_{2}\right\rangle_{\Gamma_{2}} \geq 0 .
\end{aligned}
$$

Proof. From Lemma 4.3, $\tilde{s}$ satisfies the regular point condition, then applying the Theorem 3.1 given in [5], p. 57, there exist Lagrange multipliers $\boldsymbol{\lambda}=\left(\boldsymbol{\lambda}_{1}, \lambda_{2}, \lambda_{3}, \lambda_{4}, \boldsymbol{\lambda}_{5}, \lambda_{6}, \lambda_{7}\right) \in \mathbb{Y}^{\prime}$ such that

$$
\mathcal{L}_{\boldsymbol{s}}(\tilde{\boldsymbol{s}}, \boldsymbol{\lambda}) \boldsymbol{\tau} \geq 0 \quad \forall \boldsymbol{\tau} \in \mathbb{H} \times \mathcal{C}\left(\tilde{\boldsymbol{g}}_{1}\right) \times \mathcal{C}\left(\tilde{g}_{2}\right),
$$

where $\mathcal{L}$ is the the Lagrange functional defined by $\mathcal{L}(\tilde{\boldsymbol{s}}, \boldsymbol{\lambda})=J(\tilde{\boldsymbol{s}})-\langle\boldsymbol{\lambda}, \mathbb{F}(\tilde{\boldsymbol{s}})\rangle_{\mathbb{Y}^{\prime}}$.

Therefore, taking into account the Lemma 4.1 and Lemma 4.2 the result is followed.

From Theorem 4.1 we can derive the following optimality system:

State equations

$$
\begin{aligned}
&-\nu_{1} \Delta \tilde{\boldsymbol{u}}+\tilde{\boldsymbol{u}} \cdot \nabla \tilde{\boldsymbol{u}}+\alpha \mathbf{G} \tilde{\theta}_{1}-2 \nu_{r} \operatorname{rot} \tilde{w}=\boldsymbol{f} \text { in } \mathbf{V}_{f}^{\prime}, \\
&-\nu_{2} \Delta \tilde{w}+\tilde{\boldsymbol{u}} \cdot \nabla \tilde{w}+4 \nu_{r} \tilde{w}-2 \nu_{r} \operatorname{rot} \tilde{\boldsymbol{u}}=g \text { in } H^{-1}\left(\Omega_{f}\right), \\
& \operatorname{div} \tilde{\boldsymbol{u}}=0 \quad \text { in } \Omega_{f}, \\
&-\kappa_{1} \Delta \tilde{\theta}_{1}+\tilde{\boldsymbol{u}} \cdot \nabla \tilde{\theta}_{1}=h_{1} \quad \text { in } W_{1}^{\prime}, \\
&-\kappa_{2} \Delta \tilde{\theta}_{2}=h_{2} \text { in } W_{2}^{\prime}, \\
& \tilde{\boldsymbol{u}}=\boldsymbol{g}_{1}, \quad \tilde{w}=0, \quad \tilde{\theta}_{1}=\eta_{1} \quad \text { on } \Gamma_{1}, \\
& \kappa_{1} \frac{\partial \tilde{\theta}_{1}}{\partial \boldsymbol{n}_{1}}=\tilde{g}_{2}, \quad \kappa_{2} \frac{\partial \tilde{\theta}_{2}}{\partial \boldsymbol{n}_{2}}=-\tilde{g}_{2}, \quad \tilde{\boldsymbol{u}}=\mathbf{0}, \quad \tilde{w}=0 \quad \text { on } \Gamma_{0}, \\
& \tilde{\theta}_{2}=\eta_{2} \quad \text { on } \Gamma_{2} .
\end{aligned}
$$


Adjoint equations

$$
\begin{aligned}
\nu_{1} \Delta \boldsymbol{\lambda}_{1}+\tilde{\boldsymbol{u}} \cdot \nabla \boldsymbol{\lambda}_{1}+\nabla^{T} \boldsymbol{\lambda}_{1} \cdot \tilde{\boldsymbol{u}}+\nabla^{T} \lambda_{2} \cdot \tilde{w}+\nabla^{T} \lambda_{3} \cdot \tilde{\theta}_{1}+2 \nu_{r} \operatorname{rot} \lambda_{2} & =\boldsymbol{u}_{d}-\tilde{\boldsymbol{u}} \text { in }\left(\mathbf{H}_{\sigma}^{1}\right)^{\prime}, \\
\nu_{2} \Delta \lambda_{2}+\tilde{\boldsymbol{u}} \cdot \nabla \lambda_{2}-4 \nu_{r} \lambda_{2}+2 \nu_{r} \operatorname{rot} \boldsymbol{\lambda}_{1} & =0 \text { in } H^{-1}\left(\Omega_{f}\right), \\
\kappa_{1} \Delta \lambda_{3}-\alpha \mathbf{G} \boldsymbol{\lambda}_{1}+\tilde{\boldsymbol{u}} \cdot \nabla \lambda_{3} & =0 \text { in }\left(H^{1}\left(\Omega_{f}\right)\right)^{\prime}, \\
\kappa_{2} \Delta \lambda_{4} & =0 \text { in }\left(H^{1}\left(\Omega_{s}\right)\right)^{\prime}, \\
\operatorname{div} \boldsymbol{\lambda}_{1} & =0 \text { in } \Omega_{f}, \\
\nu_{1} \frac{\partial \boldsymbol{\lambda}_{1}}{\partial \boldsymbol{n}_{1}}+\boldsymbol{\lambda}_{5} & =\mathbf{0} \quad \text { on } \Gamma_{0} \cup \Gamma_{1}, \\
\kappa_{1} \frac{\partial \lambda_{3}}{\partial \boldsymbol{n}_{1}}=\tilde{\theta}_{1}-\tilde{\theta}_{2}, \quad \kappa_{2} \frac{\partial \lambda_{4}}{\partial \boldsymbol{n}_{2}} & =\tilde{\theta}_{2}-\tilde{\theta}_{1} \quad \text { on } \Gamma_{0}, \\
\lambda_{6}=0 \quad \text { on } \Gamma_{1}, \quad \lambda_{7} & =0 \quad \text { on } \Gamma_{2} .
\end{aligned}
$$

Optimality conditions

$$
\left\langle\beta_{1} \tilde{\boldsymbol{g}}_{1}+\boldsymbol{\lambda}_{5}, \boldsymbol{g}_{1}-\tilde{\boldsymbol{g}}_{1}\right\rangle_{\Gamma_{1}} \geq 0, \quad\left\langle\beta_{2} \tilde{g}_{2}+\lambda_{3}-\lambda_{4}, g_{2}-\tilde{g}_{2}\right\rangle_{\Gamma_{0}} \geq 0 \quad \forall\left(\boldsymbol{g}_{1}, g_{2}\right) \in \mathcal{U}_{a d}
$$

\section{Conclusions}

For viscosities and thermic conductivity coefficients large enough of the fluids, we proved existence and uniqueness of weak solutions for the system (1)-(9). In order to minimize the temperature difference in the solid-liquid interface of the domain, is established a control problem associated with the weak solutions of the system (1)-(9), considering as control parameters a function defined on one part of the boundary of the fluid region and another defined on the interface of the solid-fluid region. For the control problem is proved the existence of solution and an optimality system is obtained by using the Lagrange multipliers method.

\section{References}

[1] K. Kunisch, J.C. de los Reyes. A semi-smooth Newton method for control constrained boundary optimal control of the Navier-Stokes equations, Nonlinear Analysis, 62:1289-1316, 2005.

[2] H.C. Lee, O.Y. Imanuvilov. Analysis of Neumann boundary optimal control problems for the stationary Boussinesq equations including solid media, SIAM J. Control Optim., 39:457-477, 2000.

[3] H. K. Lee. An Optimization-based Domain Decomposition Method for the Boussinesq Equations. Numer. Methods Partial Differential Equations, 18:1-25, 2002.

[4] G. Lukaszewicz. Micropolar Fluids: Theory and Applications. Birkhauser, 1999.

[5] J. Zowe, S. Kurcyusz. Regularity and Stability for the Mathematical Programming Problem in Banach Spaces, Applied Mathematics and Optimization, 5:49-62, 1979. 